\title{
Observations of warm dust near methanol masers ${ }^{\star}$
}

\author{
A. J. Walsh ${ }^{1,2}$, G. H. Macdonald ${ }^{3}$, N. D. S. Alvey ${ }^{3}$, M. G. Burton ${ }^{4}$, and J.-K. Lee ${ }^{4}$ \\ 1 Max Planck Institut für Radioastronomie, auf dem Hügel 69, 53121 Bonn, Germany \\ ${ }^{2}$ Harvard-Smithsonian Center for Astrophysics, 60 Garden Street, Cambridge, MA, 02138, USA \\ 3 Department of Electronics, University of Kent, Canterbury, Kent CT2 7NT, UK \\ ${ }^{4}$ Department of Astrophysics and Optics, School of Physics, University of New South Wales, NSW, 2052, Australia
}

Received 16 July 2001 / Accepted 29 July 2003

\begin{abstract}
Continuum emission at 450 and $850 \mu \mathrm{m}$ from warm dust has been mapped in the fields of 71 methanol masers. Within these fields lie 30 centimetre-wave radio continuum sources and an additional 13 methanol maser sites. Sub-mm emission is detected at all but one of the maser sites, confirming the association of methanol maser emission with deeply embedded objects. Measured bolometric luminosities confirm that methanol maser emission is an excellent signpost of high-mass star formation. Examples of nearby isolated maserless dust cores may be harbouring massive protostars at an earlier evolutionary stage.
\end{abstract}

Key words. stars: formation - ISM: dust, extinction - submillimeter - infrared: ISM

\section{Introduction}

It has been recognised for some time that maser action in methanol lines commonly occurs in regions of massive star formation (Menten et al. 1986). In particular, the $5_{1}-6_{0} \mathrm{~A}^{+}$transition at $6.7 \mathrm{GHz}$ is one of the strongest and most widespread interstellar masers known (Menten 1991).

Maser emission from methanol can be divided into two mutually exclusive classes: Class I and Class II. Class I masers are found in the general vicinity of massive star forming regions but are offset from indicators of high-mass star formation such as compact HII regions, strong infrared sources and $\mathrm{OH}$ masers. It has been suggested that Class I masers are associated with shock fronts at the interface between mass outflows and dense ambient material (Plambeck et al. 1990).

Conversely, Class II masers have been claimed to be closely associated with $\mathrm{OH}$ masers and ultracompact (UC) HII regions (Menten et al. 1988, 1988, 1995). However, recent work by Walsh and his associates (Walsh et al. 1997, 1998, 1999) has demonstrated that most Class II $6.7 \mathrm{GHz}$ methanol maser sources are not associated with a detectable UC HII region. This result may be interpreted in two ways: either the methanol maser turns on before the UC HII region is created and is then destroyed as the UC HII region develops (Walsh et al. 1998), or alternatively the maser is pumped by radiation from a nearby star with insufficient ionising flux to create an observable UC HII region (a star later than B3 in spectral type)

Send offprint requests to: A. J. Walsh,

e-mail: awalsh@cfa.harvard.edu

* Full version of Fig. 1 is only available in electronic form at http://www.edpsciences.org
(Phillips et al. 1998). These two hypotheses can be differentiated by measurement of the spectral energy distribution (SED) of the source of excitation.

Submillimetre emission from warm dust is an excellent tracer of column density in star formation regions because it generally remains optically thin. In this paper we present maps of the emission from warm dust near $6.7 \mathrm{GHz}$ Class II methanol masers at wavelengths of $450 \mu \mathrm{m}$ and $850 \mu \mathrm{m}$ made using the Submillimetre Common-User Bolometer Array (SCUBA) on the James Clerk Maxwell Telescope (JCMT). SCUBA has a field of view of 2.5 and resolution of $8^{\prime \prime} / 15^{\prime \prime}$ at $450 / 850 \mu \mathrm{m}$ respectively, allowing mapping of dust over several orders of magnitude of column density with a spatial resolution typically a few parsecs at the distance of the methanol masers. Unfortunately, at the time of the observations, photometry at $1350 \mu \mathrm{m}$ and $2000 \mu \mathrm{m}$ was unavailable on SCUBA.

The source sample of $6.7 \mathrm{GHz}$ methanol masers was selected from the survey of Walsh et al. (1998) in which 223 maser sites were detected from a sample of 276 IRAS sources with UC HII colours (Wood \& Churchwell 1989a). We present here SCUBA maps of the 84 methanol masers in this survey lying in the declination range $0^{\circ}>\delta>-20^{\circ}$. In this paper, we compare the spatial distributions of the submillimetre and radio continuum from the dust and ionised gas with the maser emission.

Hatchell et al. (2000) have recently published $450 / 850 \mu \mathrm{m}$ SCUBA maps of the dust emission in a small sample of highmass star forming regions for which extensive molecular line data were available. They found that although all sources showed centrally peaked emission, those with the strongest peaks and highest submillimetre fluxes were also those 
showing rich molecular line spectra characteristic of "hot molecular cores" (HMC). Following this result, we identify in our sample the most probable HMC candidates.

In summary, the motivation in mapping the submillimetre emission from warm dust in the vicinity of methanol masers is four-fold:

(i) to confirm that the maser emission arises from deeply embedded objects;

(ii) to study the relationship between small-scale structure delineated by the maser emission and radio continuum emission from ionised gas and the larger-scale structure of the dust;

(iii) to search for other condensations which may be members of the same cluster as the maser sources but represent either a hot molecular core or an earlier stage in the evolution of a massive protostar;

(iv) to determine the SED of the maser pumping source or putative massive protostellar object in the same cluster.

We stress that the main purpose of this paper is to present our images. The dust emission from these sources has been examined with detailed models by Alvey (2001). In Sect. 2 we describe the observations and procedure for data reduction. The images are discussed in Sect. 3 and our conclusions given in Sect. 4.

\section{Observations and data reduction}

Observations were made during the period 1-4 April 2000 using SCUBA on the JCMT (Holland et al. 1999) simultaneously at $450 \mu \mathrm{m}$ and $850 \mu \mathrm{m}$. SCUBA comprises two hexagonal arrays of bolometers, giving 91 pixels at $450 \mu \mathrm{m}$ and 37 pixels at $850 \mu \mathrm{m}$ and a field of view of 2.5 at each wavelength. In order to achieve fully sampled maps, SCUBA is operated in a "jiggle" mode in which the telescope is moved through a 64-position pattern by the secondary mirror so that the field is fully sampled between the more widely separated intrinsic beam positions of the array. The chop throw was 120 " in the azimuth direction and the integration time was 6 minutes per source.

The data were reduced using the SCUBA User Reduction Facility, SURF (Jenness et al. 1998). Calibration was carried out using IRAS 16293-0242 and G34.3+0.15.

The beam sizes were taken from the SCUBA web pages and correspond to integrated beam profiles of 118.5 and 315.5 square arcseconds at 450 and $850 \mu \mathrm{m}$ respectively. Assuming the JCMT diffraction beam to be approximately Gaussian with $F W H M$ of $8^{\prime \prime}$ and $15^{\prime \prime}$ at 450 and $850 \mu \mathrm{m}$ respectively we estimate that $38 \%$ of the flux at $450 \mu \mathrm{m}$ and $19 \%$ at $850 \mu \mathrm{m}$ is contained within the error beam.

Care was taken in calculating the integrated flux, since the regions we have observed tend to have high source densities and in many cases we observe strong negative features in our maps due to emission in the reference position, as well as extended emission across the field. In cases of strong negative emission interfering with the determination of a source flux, we provide only an upper limit by measuring only the positive emission.
We estimate that calibration errors are typically $\pm 40 \%$ at $450 \mu \mathrm{m}$ and $\pm 20 \%$ at $850 \mu \mathrm{m}$. These errors are mainly due to error beam pick up of sky contibutions.

\section{Results}

71 fields containing methanol maser and radio continuum emission were observed with SCUBA. Figure 1 shows the $850 \mu \mathrm{m}$ and $450 \mu \mathrm{m}$ sub-mm emission from the observed fields, together with the locations of methanol maser sites, UC HII regions and infrared sources. The names of the fields are derived from the Galactic coordinates. Individual maser, sub-mm or radio continuum sources are named using the same convention. There are 84 methanol maser sites and 30 radio continuum sources detected by Walsh et al. (1998) found within the fields. Of the 84 maser sites, $70(83 \%)$ are within 5" of a submm peak and $11(13 \%)$ are between 5 and $10^{\prime \prime}$ of a sub-mm peak. We find all but one source $(\mathrm{G} 10.10+0.73)$ within the submm emission contours. Of the 30 radio continuum sources, we find $16(53 \%)$ within $5^{\prime \prime}$ of a sub-mm peak, and $10(33 \%)$ between 5 and $10^{\prime \prime}$ of a sub-mm peak. However, all the radio continuum sources are contained within extended sub-mm continuum emission. We also provide the positions of mid-infrared (MIR) sources from the Midcourse Space eXperiment (MSX) Point Source Catalog version $1.2^{1}$ in Fig. 1.

We found that the morphology of the sub-mm continuum emission is generally similar at 450 and $850 \mu \mathrm{m}$, the major differences being the lower signal to noise for the $450 \mu \mathrm{m}$ images and the slightly better resolution at $450 \mu \mathrm{m}$. Important differences between the 450 and $850 \mu \mathrm{m}$ images are reported in Sect. 3.2.

Table 1 lists the peak flux density and integrated flux of sub-mm sources of interest found within the fields shown in Fig. 1. To calculate the integrated flux, the flux was summed over an area approximating the extent of the emission, avoiding other sources. A background value was subtracted off the integrated flux, which was determined by summing over an area in the field without strong emission. Fluxes are not calculated for sources where a significant proportion of the flux lies outside the imaged field, e.g. the northern corner of G0.21-0.00. For some sources, photometry is unreliable due to contamination from the off beam. Such sources are marked as lower limits in Table 1 . Typical $1 \sigma$ noise levels are $50 \mathrm{mJy}$ beam $^{-1}$ at $850 \mu \mathrm{m}$ and $0.5 \mathrm{Jy} \mathrm{beam}^{-1}$ at $450 \mu \mathrm{m}$.

\subsection{A maser site associated with a planetary nebula?}

G10.10+0.73 (18021-1950), shown in Figs. 1y and z, is the one methanol maser site that is not associated with any sub-mm continuum emission, suggesting it may be a peculiar source. It is even more intriguing when one considers that the field contains a radio continuum source in the north-west corner, coincident with a compact sub-mm source. This radio source is associated with the powering source of the well-known bipolar planetary nebula NGC 6537, and is thus not an UC HII region,

\footnotetext{
1 Available at http://www.ipac.caltech.edu/ipac/msx/ msx.html
} 

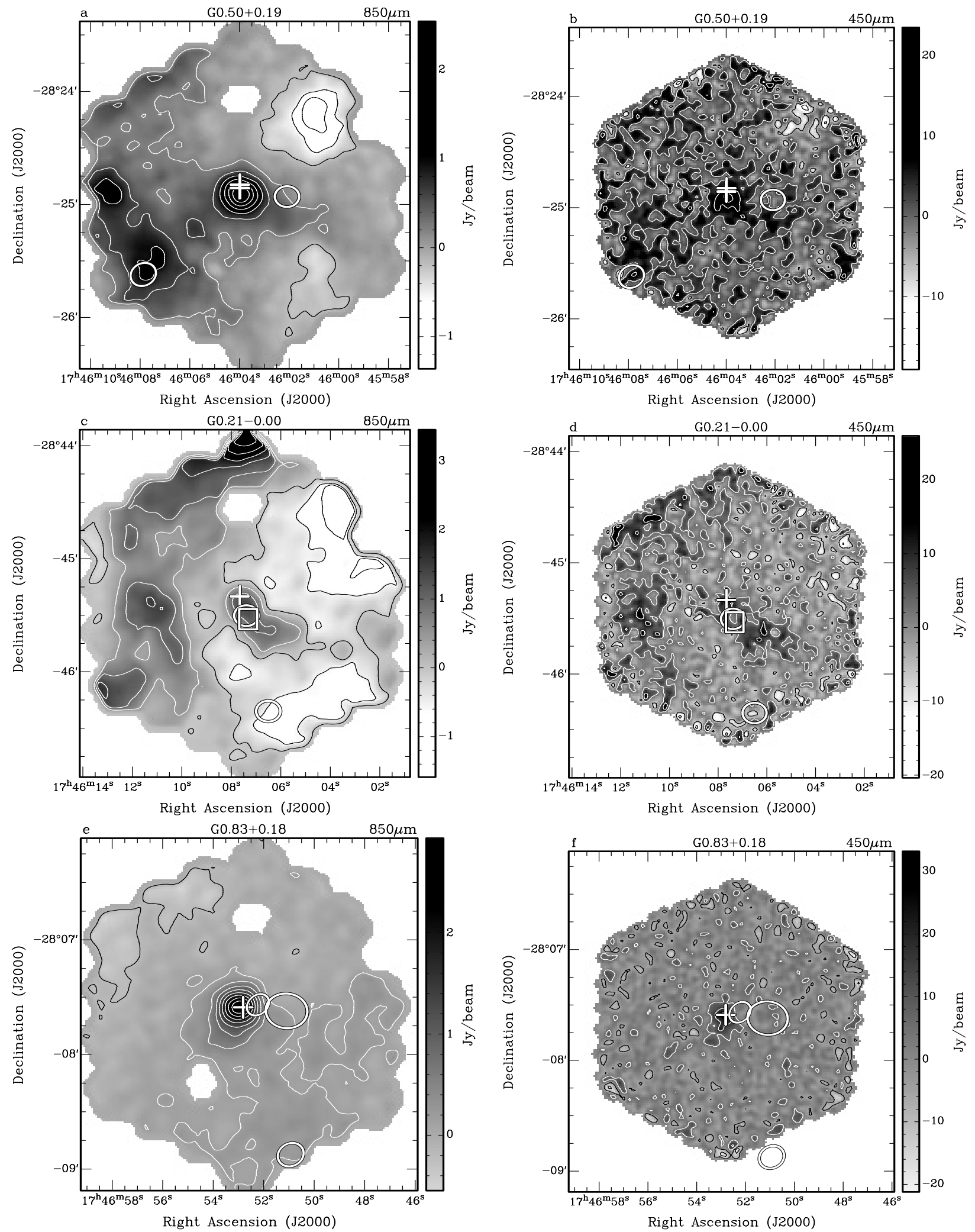

Fig. 1. a)-f) $850 \mu \mathrm{m}$ greyscale and contour maps of the observed fields. In each case the contours are 10, 20, ..., 90\% of the peak level. Plus symbols represent the positions of methanol maser sites and squares represent the positions of UC HII regions (Walsh et al. 1998). Ellipses represent the position of MSX sources from the Point Source Catalog, version 1.2, with the size of the ellipse five times the $1 \sigma$ MSX position error. Bad bolometer pixels, which have been removed show up as white "holes" in the fields. For example Fig. 1d, to the north of the main source. 
Table 1. Sub-mm source characteristics. Column 1 lists the field name. Suffixes of A, B and C are added to the name for multiple sources in each field, with the brightest named first. Source coordinates are given in Cols. 2 and 3. Column 4 lists the peak flux density at $850 \mu \mathrm{m}$ and Col. 5 lists (where available) the integrated flux over an aperture centred on the source coordinates. The $450 \mu \mathrm{m}$ peak flux is listed in Col. 6 , with the integrated flux listed in Col. 7. Column 8 lists whether the source is associated with a methanol maser site (m), a radio continuum source (r) or both (mr).

\begin{tabular}{|c|c|c|c|c|c|c|c|}
\hline \multirow{3}{*}{$\begin{array}{l}\text { Source } \\
\text { name }\end{array}$} & \multirow{2}{*}{\multicolumn{2}{|c|}{ Coordinates }} & \multicolumn{2}{|c|}{$850 \mu \mathrm{m}$} & \multicolumn{2}{|c|}{$450 \mu \mathrm{m}$} & \multirow[t]{3}{*}{ Assoc } \\
\hline & & & \multirow{2}{*}{$\begin{array}{c}\text { Peak flux } \\
\text { density } \\
\text { (Jy/beam) }\end{array}$} & \multirow{2}{*}{$\begin{array}{l}\text { Int. } \\
\text { flux } \\
(\mathrm{Jy})\end{array}$} & \multirow{2}{*}{$\begin{array}{c}\text { Peak flux } \\
\text { density } \\
(\mathrm{Jy} / \text { beam })\end{array}$} & \multirow{2}{*}{$\begin{array}{l}\text { Int. } \\
\text { flux } \\
(\mathrm{Jy})\end{array}$} & \\
\hline & $\begin{array}{c}\text { RA } \\
(\mathrm{J} 2000)\end{array}$ & $\begin{array}{c}\text { Dec } \\
(\mathrm{J} 2000)\end{array}$ & & & & & \\
\hline G0.50+0.19 & 174603.9 & -282458 & 2.5 & 4.0 & 23 & 57 & $\mathrm{~m}$ \\
\hline G0.21-0.00 & 174607.6 & -284521 & 1.4 & 6.2 & 16 & 98 & $\mathrm{mr}$ \\
\hline G0.83+0.18 & 174652.8 & -280735 & 2.9 & 4.2 & 33 & 64 & $\mathrm{~m}$ \\
\hline G0.32-0.20 & 174709.1 & -284617 & 5.8 & 20 & 56 & 320 & $\mathrm{mr}$ \\
\hline G1.15-0.12 & 174848.9 & -280113 & 1.5 & 1.9 & 12 & 31 & $\mathrm{~m}$ \\
\hline G0.55-0.85 & 175014.2 & -285430 & 24 & 52 & 180 & 850 & $\mathrm{~m}$ \\
\hline $\mathrm{G} 2.54+0.20$ & 175046.8 & -263946 & 2.1 & 12 & 17 & 150 & $\mathrm{~m}$ \\
\hline G5.90-0.43 & 180040.7 & -240423 & 14 & 33 & 94 & 470 & $\mathrm{~m}$ \\
\hline G6.54-0.11A & 180051.4 & -232136 & 0.6 & 0.8 & 8.6 & 16 & $\mathrm{~m}$ \\
\hline G6.54-0.11B & 180050.1 & -232040 & 2.4 & 8.6 & 7.0 & 93 & $\mathrm{r}$ \\
\hline G6.61-0.08 & 180054.2 & -231702 & 0.86 & 1.1 & 9.7 & 11 & $\mathrm{~m}$ \\
\hline G8.14+0.23A & 180302.5 & -214804 & 4.9 & 8.0 & 40 & 110 & \\
\hline G8.14+0.23B & 180301.7 & -214815 & 5.3 & 11 & 40 & 130 & $\mathrm{mr}$ \\
\hline G9.62+0.19 & 180414.8 & -203136 & 12 & 30 & 73 & 400 & $\mathrm{mr}$ \\
\hline $\mathrm{G} 10.10+0.73$ & 180513.2 & -195037 & 0.9 & 1.4 & 8.5 & 13 & $\mathrm{r}$ \\
\hline G8.68-0.36A & 180619.2 & -213732 & 16 & 42 & 110 & 730 & $\mathrm{mr}$ \\
\hline G8.68-0.36B & 180623.5 & -213710 & 5.1 & 14 & 48 & 230 & $\mathrm{~m}$ \\
\hline G9.99-0.03A & 180750.4 & -201857 & 2.5 & 3.8 & 23 & 66 & $\mathrm{~m}$ \\
\hline G9.99-0.03B & 180753.3 & -201827 & 0.79 & 0.9 & 7.4 & 17 & $\mathrm{r}$ \\
\hline $\mathrm{G} 10.47+0.03 \mathrm{~A}$ & 180838.5 & -195152 & 39 & 59 & 200 & 660 & $\mathrm{mr}$ \\
\hline G10.47+0.03B & 180836.7 & -195214 & 6.2 & 9.7 & 33 & 140 & $\mathrm{r}$ \\
\hline $\mathrm{G} 10.47+0.03 \mathrm{C}$ & 180838.1 & -195114 & 4.2 & 8.4 & 29 & 170 & $\mathrm{~m}$ \\
\hline G10.44-0.02 & 180845.0 & -195441 & 2.2 & 6.8 & 16 & 94 & $\mathrm{~m}$ \\
\hline G10.29-0.13A & 180849.4 & -200602 & 4.7 & 11 & 37 & 210 & $\mathrm{~m}$ \\
\hline G10.29-0.13B & 180846.9 & -200551 & 3.5 & 17 & 23 & 340 & \\
\hline G10.29-0.13C & 180852.6 & -200608 & 2.5 & 7.6 & 22 & 110 & \\
\hline G10.30-0.15A & 180856.1 & -200557 & 6.2 & 26 & 52 & 550 & $\mathrm{mr}$ \\
\hline G10.30-0.15B & 180852.6 & -200609 & 2.6 & 7.7 & 24 & 140 & \\
\hline G10.34-0.14A & 180900.1 & -200339 & 5.3 & 15 & 47 & 220 & $\mathrm{~m}$ \\
\hline G10.34-0.14B & 180903.1 & -200303 & 1.9 & 7.3 & 11 & 85 & \\
\hline G10.32-0.16 & 180901.6 & -200511 & $\geq 3.7$ & & $\geq 29$ & & $\mathrm{~m}$ \\
\hline G10.63-0.38 & 181028.7 & -195552 & 34 & 76 & 220 & 1100 & $\mathrm{mr}$ \\
\hline G10.63-0.33A & 181018.7 & -195428 & $\geq 2.5$ & & $\geq 18$ & & $\mathrm{~m}$ \\
\hline G10.63-0.33B & 181015.7 & -195445 & 1.8 & 4.5 & 9.1 & 45 & \\
\hline G12.89+0.49 & 181151.4 & -173131 & 11 & 19 & 82 & 280 & $\mathrm{~m}$ \\
\hline G12.03-0.03 & 181201.7 & -183158 & 1.3 & 2.9 & 9.4 & 23 & $\mathrm{~m}$ \\
\hline G11.94-0.15A & 181219.5 & -183953 & $\geq 1.5$ & & 11 & 29 & \\
\hline G11.94-0.15B & 181218.2 & -184005 & 1.4 & 2.5 & 6.5 & 19 & $\mathrm{~m}$ \\
\hline G12.21-0.09A & 181239.8 & -182418 & 12 & 17 & 95 & 270 & $\mathrm{mr}$ \\
\hline G12.21-0.09B & 181240.4 & -182446 & 1.3 & & 30 & & $\mathrm{~m}$ \\
\hline G12.21-0.09C & 181237.7 & -182409 & 0.4 & & 8.1 & & $\mathrm{~m}$ \\
\hline G12.18-0.12A & 181242.7 & -182508 & $\geq 2.0$ & & $\geq 7.1$ & & $\mathrm{~m}$ \\
\hline G12.18-0.12B & 181241.0 & -182617 & 0.73 & 1.0 & 3.9 & 13 & $\mathrm{~m}$ \\
\hline G11.99-0.27 & 181251.2 & -184037 & 1.0 & 1.7 & 9.7 & 22 & $\mathrm{~m}$ \\
\hline G12.68-0.18A & 181354.7 & -180150 & 5.0 & 11 & 31 & 73 & $\mathrm{~m}$ \\
\hline G12.68-0.18B & 181355.5 & -180127 & 2.0 & 3.6 & 14 & 42 & \\
\hline G11.94-0.62A & 181400.9 & -185326 & 7.4 & 22 & 46 & 380 & $\mathrm{mr}$ \\
\hline G11.94-0.62B & 181357.9 & -185420 & 4.6 & 12 & 25 & 140 & \\
\hline G12.91-0.26 & 181439.5 & -175203 & 8.9 & 25 & 51 & 300 & $\mathrm{~m}$ \\
\hline G11.50-1.49 & 181622.3 & -194127 & 5.1 & 19 & 42 & 210 & $\mathrm{~m}$ \\
\hline
\end{tabular}


Table 1. continued.

\begin{tabular}{|c|c|c|c|c|c|c|c|}
\hline \multirow{3}{*}{$\begin{array}{l}\text { Source } \\
\text { name }\end{array}$} & & & \multicolumn{2}{|c|}{$850 \mu \mathrm{m}$} & \multicolumn{2}{|c|}{$450 \mu \mathrm{m}$} & \multirow[t]{3}{*}{ Assoc. } \\
\hline & \multicolumn{2}{|c|}{ Coordinates } & Peak flux & Int. & Peak flux & Int. & \\
\hline & $\begin{array}{c}\text { RA } \\
(\mathrm{J} 2000)\end{array}$ & $\begin{array}{c}\text { Dec } \\
(\mathrm{J} 2000)\end{array}$ & $\begin{array}{c}\text { density } \\
\text { (Jy/beam) }\end{array}$ & $\begin{array}{l}\text { flux } \\
\text { (Jy) }\end{array}$ & $\begin{array}{c}\text { density } \\
\text { (Jy/beam) }\end{array}$ & $\begin{array}{l}\text { flux } \\
\text { (Jy) }\end{array}$ & \\
\hline $\mathrm{G} 14.60+0.02 \mathrm{~A}$ & 181702.7 & -161424 & 2.7 & 4.8 & 39 & 78 & \\
\hline $\mathrm{G} 14.60+0.02 \mathrm{~B}$ & 181701.9 & -161435 & 2.1 & 3.4 & 28 & 74 & $\mathrm{~m}$ \\
\hline $\mathrm{G} 14.60+0.02 \mathrm{C}$ & 181700.5 & -161449 & 1.1 & 1.4 & 16 & 24 & $\mathrm{r}$ \\
\hline G15.03-0.68A & 182023.1 & -161153 & 17 & 31 & 130 & 790 & \\
\hline G15.03-0.68B & 182024.9 & -161133 & 15 & 16 & 100 & 270 & $\mathrm{~m}$ \\
\hline G15.03-0.68C & 182022.6 & -161121 & 19 & 37 & 120 & 820 & \\
\hline G16.59+0.15 & 182109.3 & -143149 & 7.2 & 14 & 65 & 270 & $\mathrm{~m}$ \\
\hline $\mathrm{G} 19.47+0.17$ & 182554.7 & -115232 & 7.8 & 17 & 72 & 380 & $\mathrm{~m}$ \\
\hline G19.49+0.15 & 182600.5 & -115220 & 1.5 & 3.3 & 13 & 47 & $\mathrm{~m}$ \\
\hline G19.36-0.03 & 182625.9 & -120354 & 3.8 & 11 & 29 & 210 & $\mathrm{~m}$ \\
\hline G19.61-0.13A & 182717.1 & -115353 & 2.8 & 9.4 & 20 & 160 & $\mathrm{~m}$ \\
\hline G19.61-0.13B & 182713.7 & -115321 & 1.4 & 3.0 & 9.9 & 48 & $\mathrm{~m}$ \\
\hline G19.70-0.27A & 182755.3 & -115240 & 2.1 & 2.9 & 16 & 37 & $\mathrm{~m}$ \\
\hline G19.70-0.27B & 182753.8 & -115227 & 1.2 & 2.0 & 7.4 & 31 & \\
\hline G16.86-2.16A & 182924.2 & -151606 & 8.6 & 16 & 48 & 180 & $\mathrm{~m}$ \\
\hline G16.86-2.16B & 182924.7 & -151525 & 6.2 & 33 & 30 & 470 & \\
\hline $\mathrm{G} 21.88+0.01 \mathrm{~A}$ & 183102.3 & -094927 & 1.5 & 4.0 & 7.3 & 40 & $\mathrm{r}$ \\
\hline $\mathrm{G} 21.88+0.01 \mathrm{~B}$ & 183101.6 & -094859 & 1.0 & 1.3 & 7.7 & 17 & $\mathrm{~m}$ \\
\hline $\mathrm{G} 22.36+0.07 \mathrm{~A}$ & 183143.9 & -092211 & 1.7 & 1.8 & 10 & 18 & $\mathrm{~m}$ \\
\hline $\mathrm{G} 22.36+0.07 \mathrm{~B}$ & 183143.2 & -092225 & 1.8 & 2.7 & 8.3 & 33 & \\
\hline $\mathrm{G} 25.65+1.05$ & 183421.1 & -055944 & 9.2 & 25 & 49 & 220 & $\mathrm{mr}$ \\
\hline G23.26-0.24 & 183431.6 & -084248 & 1.4 & 2.5 & 6.1 & 39 & $\mathrm{~m}$ \\
\hline G23.44-0.18 & 183439.4 & -083140 & 6.7 & 18 & 36 & 200 & $\mathrm{~m}$ \\
\hline G24.79+0.08 & 183612.6 & -071214 & 16 & 34 & 93 & 400 & $\mathrm{~m}$ \\
\hline $\mathrm{G} 24.85+0.09$ & 183618.5 & -070855 & 1.5 & 3.3 & 9.9 & 34 & $\mathrm{~m}$ \\
\hline $\mathrm{G} 25.71+0.04 \mathrm{~A}$ & 183803.2 & -062418 & 2.3 & 3.8 & 21 & 65 & $\mathrm{~m}$ \\
\hline $\mathrm{G} 25.71+0.04 \mathrm{~B}$ & 183802.8 & -062353 & 1.1 & 1.5 & 7.0 & 38 & $\mathrm{r}$ \\
\hline G25.83-0.18 & 183903.4 & -062413 & 10 & 16 & 60 & 160 & $\mathrm{~m}$ \\
\hline $\mathrm{G} 28.15-0.00$ & 184242.6 & -041535 & 1.9 & 4.6 & 8.6 & 35 & $\mathrm{~m}$ \\
\hline G28.20-0.05 & 184258.1 & -041359 & 9.7 & 25 & 57 & 300 & $\mathrm{mr}$ \\
\hline $\mathrm{G} 28.28-0.36 \mathrm{~A}$ & 184414.8 & -041744 & 2.0 & 5.4 & 9.5 & 55 & $\mathrm{r}$ \\
\hline G28.28-0.36B & 184414.1 & -041804 & 1.8 & 5.1 & 8.2 & 58 & $\mathrm{~m}$ \\
\hline G28.31-0.39A & 184420.5 & -041743 & 1.2 & 2.0 & 5.2 & 20 & \\
\hline $\mathrm{G} 28.31-0.39 \mathrm{~B}$ & 184422.0 & -041741 & 1.0 & 2.0 & 3.9 & 14 & $\mathrm{~m}$ \\
\hline G28.85-0.23 & 184447.0 & -034422 & $\geq 0.4$ & & $\geq 0.9$ & & $\mathrm{~m}$ \\
\hline G28.83-0.25 & 184451.1 & -034549 & 4.8 & 12 & 30 & 150 & $\mathrm{~m}$ \\
\hline G29.86-0.04 & 184559.7 & -024509 & 1.9 & 4.8 & 13 & 63 & $\mathrm{~m}$ \\
\hline G29.96-0.02 & 184603.9 & -023923 & 13 & 26 & 81 & 390 & $\mathrm{mr}$ \\
\hline G29.98-0.05 & 184613.1 & -023904 & 2.6 & 9.3 & 14 & 59 & $\mathrm{~m}$ \\
\hline $\mathrm{G} 30.82+0.27$ & 184636.4 & -014521 & 0.8 & 2.7 & 4.9 & 32 & $\mathrm{~m}$ \\
\hline $\mathrm{G} 30.78+0.23$ & 184641.4 & -014840 & $\geq 0.6$ & & $\geq 4.0$ & & $\mathrm{~m}$ \\
\hline $\mathrm{G} 30.79+0.20$ & 184648.0 & -014854 & 3.6 & 8.7 & 20 & 90 & $\mathrm{~m}$ \\
\hline G30.90+0.16 & 184709.1 & -014413 & 2.4 & 6.2 & 16 & 56 & $\mathrm{~m}$ \\
\hline G30.59-0.04 & 184718.8 & -020618 & 4.0 & 12 & 27 & 113 & $\mathrm{~m}$ \\
\hline $\mathrm{G} 31.41+0.31$ & 184734.3 & -011247 & 26 & 40 & 140 & 560 & $\mathrm{mr}$ \\
\hline G30.71-0.06 & 184736.7 & -020053 & 14 & 29 & 110 & 460 & $\mathrm{~m}$ \\
\hline G30.76-0.05A & 184738.4 & -015744 & 4.0 & 6.7 & 30 & 79 & \\
\hline G30.76-0.05B & 184739.5 & -015725 & 2.0 & 2.4 & 13 & 41 & $\mathrm{~m}$ \\
\hline G31.06+0.09 & 184741.5 & -013730 & 1.0 & 1.8 & $<5$ & & $\mathrm{~m}$ \\
\hline G30.82-0.05 & 184746.7 & -015427 & 26 & 54 & 160 & 610 & $\mathrm{~m}$ \\
\hline $\mathrm{G} 31.28+0.06$ & 184812.3 & -012631 & 6.8 & 15 & 48 & 170 & $\mathrm{mr}$ \\
\hline
\end{tabular}

${ }^{a}$ Non-detection: the value quoted is the $3 \sigma$ upper limit. 
formed by massive star formation. The methanol maser site is found in the south-eastern corner of Fig. 1w, about 90" from the radio continuum source and thus does not overlap with the planetary nebula, which is oriented in the NE-SW direction. No sub-mm emission is observed associated with the maser site. It is not clear whether the maser site is associated with the planetary nebula or if it is just a chance alignment. The kinematics of NGC 6537 and the maser site are well matched, with $V_{\text {LSR }}$ equal to $-4.1 \mathrm{~km} \mathrm{~s}^{-1}$ for NGC 6537 (Maciel \& Dutra 1992) and the methanol maser emission $1.2 \mathrm{~km} \mathrm{~s}^{-1}$. At the very least, it is an interesting coincidence that the only maser site in our survey without detected sub-mm emission is also the only one close to a planetary nebula. It is possible that the maser site is located on the far side of the Galaxy (i.e. a far kinematic distance corresponding to $16.0 \mathrm{kpc}$ ), whereas NGC 6537 is only $0.7 \mathrm{kpc}$ away (Maciel \& Dutra 1992). However, even at this distance, a sub-mm source below our detection limit and coincident with this maser site would still have to be the weakest of all sub-mm sources coincident with maser sites in this survey. Furthermore, given an angular height off the Galactic plane of 0.73 degrees, the maser site would be about $200 \mathrm{pc}$ from the Galactic plane at the far kinematic distance and so we consider the near kinematic distance more likely. Thus, it is a target worthy of further investigation as it may be the first methanol maser site associated with an astrophysical object other than massive star formation.

\subsection{Details of individual sources ${ }^{2}$}

Here we list details of each field (except for G10.10+0.73, which is detailed above), paying particular attention to the positions of methanol maser sites and UC HII regions given by Walsh et al. (1998). The source names are derived from the Galactic coordinates of the pointing centres.

G0.50+0.19 (17429-2823) This field (shown in Figs. 1a and b) contains two methanol maser sites, separated by $2^{\prime \prime}$. Caswell (1998) reports $1.665 \mathrm{GHz} \mathrm{OH}$ maser emission close to the methanol sites. A strong, unresolved sub-mm source is found coincident with the maser sites.

G0.21-0.00 (17430-2844) This field (shown in Figs. 1c and d) contains a maser site as well as an UC HII region, separated by $10^{\prime \prime}$. Both maser site and UC HII region are coincident with sub-mm continuum emission, but the maser site is associated with stronger sub-mm emission. Strong sub-mm emission is noticed at the top of Fig. 1c. It arises from G0.32-0.20 (Fig. 1g).

G0.83+0.18 (17436-2807) This field (shown in Figs. 1e and f) has a bright, unresolved sub-mm source, found coincident with a methanol maser site.

G0.32-0.20 (17439-2845) This field (shown in Fig. 1g and $\mathrm{h}$ ) is listed as a misclassified planetary nebula (Acker et al. 1987). Two UC HII regions are found within the observed field. One is associated with a maser site and is also the brighter sub-mm source.

\footnotetext{
${ }^{2}$ Images for only the first three regions are shown in Fig. 1. All images can be found in the electronic version of this paper.
}

G1.15-0.12 (17455-2800) This field (shown in Fig. 1i and j) has a weak sub-mm source coincident with a maser site. Stronger sub-mm emission is noted in the western edge of the field. This may be associated with an UC HII region located at $174841.67-280138.7$ (J2000).

G0.55-0.85 (17470-2853) This field (shown in Figs. 1k and 1) contains a complex radio continuum source, comprised of a bright unresolved source (close to a methanol maser site) and extended emission to the north-west (covering about 15"). The extended radio continuum emission also contains one methanol maser spot. Figures $1 \mathrm{k}$ and 1 show sub-mm emission with a similar morphology as the radio continuum emission, although the sub-mm emission appears to be more extended, especially to the south-east. OH maser emission is found coincident with the methanol maser site (Forster \& Caswell 1999). The morphology of the $\mathrm{OH}$ maser site appears to be extended in the NW-SE direction, parallel to a line connecting the unresolved radio continuum source and the extended radio emission. Forster \& Caswell (1999) also report a water maser site between the $\mathrm{OH}$ and methanol maser sites and the extended radio emission.

G2.54+0.20 (17480-2636) This field (shown in Figs. 1m and $\mathrm{n}$ ) exhibits extended sub-mm emission peaking at the position of a methanol maser site. A second sub-mm source is found to the west. The two sources are so close together, it is impossible to separate their integrated fluxes, which therefore are not given in Table 1 .

G5.90-0.43 (17574-2403) This field (shown in Figs. 1o and p) contains extended sub-mm emission, with a compact, bright source coincident with the methanol maser site.

G6.54-0.11 (17577-2320a) This field (shown in Figs. 1q and r) contains strong sub-mm emission that is coincident with an UC HII region in the northern half of the field. A maser site at the centre of the field is coincident with a weak sub-mm source.

G6.61-0.08 (17577-2320b) This field (shown in Figs. 1s and t) has extended sub-mm emission which is seen throughout the observed region. The methanol maser site at the centre of the field is coincident with a local maximum in the sub-mm continuum emission.

G8.14+0.23 (17599-2148) This field (shown in Figs. 1u and v) contains an UC HII region and maser site separated by about $10^{\prime \prime}$. Both appear to be coincident with sub-mm emission, although it is not clear from the $850 \mu \mathrm{m}$ image (Fig. 1u) whether a sub-mm source is associated with each of the UC HII region and maser site, or whether there is one extended sub-mm source between the two. The $450 \mu \mathrm{m}$ image (Fig. 1v) indicates the extended emission breaks up into at least three peaks: one coincident with the maser site, one coincident with the radio continuum source and the other about $15^{\prime \prime}$ to the $\mathrm{N}-\mathrm{E}$ of the radio continuum source.

G9.62+0.19 (18032-2032) This field (shown in Figs. 1w and $\mathrm{x}$ ) has a bright, extended sub-mm source, coincident with both maser sites found in the field. The resolution of the $850 \mu \mathrm{m}$ SCUBA image shown in Fig. 1y is insufficient to separate two sub-mm sources in this field, however, two peaks are seen coincident with the two maser sites in the $450 \mu \mathrm{m}$ SCUBA map (Fig. 1z). Hofner et al. (1996) have studied this region in detail. 
The reader is referred to that paper and Hofner et al. (2001) for a more complete description of the region. Here we point out that the two sub-mm peaks, coinciding with the two maser sites are known as sources E and F by Hofner et al. (1996). Source F is the more interesting object as it shows signs of the hot core tracer $\mathrm{NH}_{3}(4,4)$ (Cesaroni et al. 1994).

G8.68-0.36 (18032-2137) This field (shown in Figs. 1aa and $a b)$ contains two maser sites. Both are coincident with compact sub-mm sources. The maser site coincident with the brighter sub-mm source is also coincident with an UC HII region. Jenness et al. (1995) have also imaged this region at 450 and $850 \mu \mathrm{m}$. They find a similar distribution of sub-mm emission, with fluxes that are within $30 \%$ of our values.

G9.99-0.03 (18048-2019) This field (shown in Figs. 1ac and ad) contains a maser site coincident with a bright compact sub-mm source. An unresolved UC HII region (45" to the north-west) is coincident with a weaker, compact sub-mm source.

G10.47+0.03 (18056-1952) This field (shown in Figs. 1ae and af) has been thoroughly investigated by Hatchell et al. (2000). Here we refer the reader to Hatchell et al. for a more detailed overview, but we mention that Figs. 1ae and af show methanol maser emission at the northern and central sub-mm cores. UC HII regions are found at the central and to the west of (i.e. not coincident with) the south-western sub-mm core.

G10.44-0.02 (18056-1954) This field (shown in Figs. 1ag and ah) has a north-south extended sub-mm source with the strongest peak coincident with a methanol maser site. Walsh et al. (1998) reports the maser site has a linear extension oriented in the NW-SE direction.

G10.30-0.15 (18060-2005a) This field (shown in Figs. 1ai and aj) exhibits strong sub-mm emission coincident with the methanol maser site and UC HII region. Extended emission is noticed in the east-west direction. It appears that there may be two unresolved sub-mm sources, corresponding to the maser site and UC HII region, which are separated by 6". Sub-mm emission coincident with the maser site located at G10.29-0.13 is seen at the far-western edge of the field, but is better represented in Figs. 1ao and ap.

G10.34-0.14 (18060-2005b) This field (shown in Figs. 1ak and al) contains a methanol maser site coincident with a compact sub-mm source.

G10.32-0.16 (18060-2005c) This field (shown in Figs. $1 \mathrm{am}$ and an) has an extended sub-mm source peaking at the position of a maser site. Sub-mm emission is also noticed at the south-west edge of the field, which originates from the source at G10.30-0.15 (Figs. 1ai and aj).

G10.29-0.13 (18060-2005d) This field (shown in Figs. 1ao and ap) has extended emission (in the east-west direction) which is seen to break up into 4 distinct clumps. The eastern-most clump is associated with G10.30-0.15 (Figs. 1ai and aj). The clump in the centre of the field is coincident with the methanol maser site. Walsh et al. (1998) noted the maser site has a linear extension in the north-south direction, perpendicular to the extended sub-mm emission.

G10.63-0.38 (18075-1956a) This field (shown in Figs. 1aq and ar) has a bright, extended sub-mm source coincident with an UC HII region. A methanol maser site is found within the sub-mm emission contours, $13^{\prime \prime}$ to the north-east of the UC HII region. It is interesting to note that Hofner \& Churchwell (1996) report water maser emission coincident with the UC HII region, showing a linear feature in the NW-SE direction. The methanol maser site, shows a similar orientation, even though it is offset from the water maser/UC Hir region source.

G10.63-0.33 (18075-1956b) This field (shown in Figs. 1as and at) has a methanol maser site which is found within the sub-mm emission contours of an extended sub$\mathrm{mm}$ source. The $450 \mu \mathrm{m}$ image (Fig. 1at) has a local peak within $4^{\prime \prime}$ of the maser site, which is not obvious in the $850 \mu \mathrm{m}$ image (Fig. 1as). A strong negative artefact is seen at the western edge of Figs. 1as and at, which is due to G10.63-0.38.

G12.89+0.49 (18089-1732) This field (shown in Figs. 1au and av) has a bright, extended sub-mm source peaking at the position of a methanol maser site. The methanol maser site is also coincident with 1.665 and $1.667 \mathrm{GHz} \mathrm{OH}$ maser emission (Caswell 1998).

G12.03-0.03 (18090-1832) This field (shown in Figs. 1aw and ax) has two sub-mm sources. The brightest one is located at the centre of the field and is coincident with the methanol maser site. Caswell (1998) reports $1.665 \mathrm{GHz}$ OH maser emission between the two sub-mm sources.

G11.94-0.15 (18094-1840) This field (shown in Figs. 1ay and az) has two sub-mm sources. The central source is coincident with the methanol maser site. The second sub-mm source is found $60^{\prime \prime}$ to the south-west of the main feature.

G12.21-0.09 (18097-1825Aa) This field (shown in Figs. 1ba and bb) contains extensive sub-mm emission and three methanol maser sites, one close to (within $5^{\prime \prime}$ ) an UC HII region. The methanol maser site close to the UC HII region is also coincident with the brightest peak in the sub-mm emission. Furthermore, the peak of the sub-mm emission is closer to the maser site than the UC HII region. This suggests there may be two sub-mm sources; one associated with the maser site and one with the UC HII region. If this is the case then the sub-mm source associated with the maser site must be the brightest. This methanol maser site is also coincident with a water maser site (Hofner \& Churchwell 1996). Both of the other methanol maser sites are coincident with a local peak in the sub-mm emission. Hatchell et al. (2000) have also mapped this field with SCUBA and report further literature details. Our maps for this and the following source (G12.18-0.13) show a similar morphology as the $350 \mu \mathrm{m}$ map of 18097-1825 taken by Hunter et al. (2000). However, Hunter et al. did not detect the weaker sub-mm sources such as G12.21-0.09B, G12.21-0.09C and G12.18-0.13B.

G12.18-0.13 (18097-1825Ab) This field (shown in Figs. $1 \mathrm{bc}$ and bd) is located about $90^{\prime \prime}$ to the south of G12.21-0.09. It contains two methanol maser sites. One is coincident with the strongest sub-mm peak, found in the northern corner of Fig. 1bc, the other is found coincident with a local peak in the sub-mm emission, at the centre of the image.

G11.99-0.27 (18099-1841) This field (shown in Figs. 1be and bf) contains one methanol maser site coincident with the sub-mm continuum peak. The sub-mm emission is extended 
around this position, with a second source being located to the west of the main peak.

G11.94-0.62 (18110-1854) This field (shown in Figs. 1bg and bh) contains a methanol maser site and UC HII region separated by $3^{\prime \prime}$. Strong sub-mm continuum emission peaks at this position, although the resolution of SCUBA is insufficient to tell whether the sub-mm emission comes from either or both of the maser site and UC HII region. Water maser emission is also found at the methanol maser site (Hofner \& Churchwell 1996). The UC HiI region shows a cometary morphology, with the head pointing towards the maser site (Hofner \& Churchwell 1996). A weaker sub-mm source is found to the south-west of the main feature.

G12.68-0.18(18117-1753a) This field (shown in Figs. 1bi and bj) contains one methanol maser site. The maser site is close to, but not coincident with, the peak of an extended sub$\mathrm{mm}$ source. The sub-mm source appears to be extended in the NE-SW direction, perpendicular to the orientation of the methanol maser site. $\mathrm{OH}$ and water maser emission is found coincident with the peak of the sub-mm emission (Forster \& Caswell 1999).

G12.91-0.26 (18117-1753b) This field (shown in Figs. 1bk and bl) contains a methanol maser site with a linear orientation in the NW-SE direction and is coincident with the peak of a bright, extended sub-mm source. The methanol maser site is coincident with 1.665 and $1.667 \mathrm{GHz} \mathrm{OH}$ (Caswell 1998) and $22 \mathrm{GHz}$ water (Forster \& Caswell 2000) maser emission.

G11.50-1.49(18134-1942) This field (shown in Figs. 1bm and bn) contains a methanol maser site coincident with the peak of an extended sub-mm source.

G14.60+0.02 (18141-1615) This field (shown in Figs. 1 bo and bp) contains an extended sub-mm source. It appears to comprise of at least three peaks. The second brightest is $10^{\prime \prime}$ from a methanol maser site. To the south-west of the maser site is an UC HII region, which is coincident with a weaker sub-mm peak.

G15.03-0.68 (18174-1612) This field (shown in Figs. 1bq and br) shows extended sub-mm emission covering most of the field. It is associated with the M 17 southern bar region. The sub-mm emission ridge overlaps the ionisation front and photodissociation region here. A methanol maser site is found at the centre of the field and is close to the peak of a weaker sub$\mathrm{mm}$ source. $\mathrm{OH}$ maser emission is reported coincident with the methanol site (Forster \& Caswell 1999). An UC HII region is found $58^{\prime \prime}$ to the north-west. While it is within the emission contours of the sub-mm continuum, it does not appear to be associated with a local peak in the sub-mm emission. The two brightest sub-mm features are found about 30 " to the north-west and south-west of the methanol maser site. Each of them is coincident with a water maser site (Forster \& Caswell 1999). A larger area around this field has been surveyed in the $\mathrm{mm}$ and sub-mm region by Hobson et al. (1993), which clearly shows the positions of the water maser sites being coincident with the sub-mm peaks.

G16.59-0.05 (18182-1433) This field (shown in Figs. 1bs and bt) shows an extended source whose peak coincides with a methanol maser site. The maser site is also coincident with water and $\mathrm{OH}$ maser sites (Forster \& Caswell 1999).

G19.49+0.15 (18232-1154a) This field (shown in Figs. 1bu and bv) shows a methanol maser site at the centre, which is coincident with the peak of an extended sub-mm feature. The sub-mm feature is extended towards the ESE, perpendicular to the orientation of the linear maser site. An UC HII region is found towards the eastern edge of the field. It is within the sub-mm emission contours, but does not appear to be coincident with a local sub-mm peak. The western edge shows bright emission which comes from G19.47+0.17 and is better shown in Figs. 1bw and bx.

G19.47+0.17 (18232-1154b) This field (shown in Figs. 1bw and bx) contains a methanol maser site coincident with the peak of a strong sub-mm source.

G19.36-0.03 (18236-1205) This field (shown in Figs. 1by and bz) exhibits extended sub-mm continuum emission. The peak of the emission coincides with a methanol maser site.

G19.61-0.13 (18244-1155) This field (shown in Figs. 1ca and $\mathrm{cb}$ ) contains two methanol maser sites, each close to, but not coincident with, the peak of a sub-mm source. The methanol maser site in the centre of the field is associated with an extended sub-mm source, while the other maser site is associated with an unresolved sub-mm source.

G19.70-0.27 (18248-1158) This field (shown in Figs. 1cc and cd) contains a methanol maser site at the centre. It is coincident with the peak of a small sub-mm source. A second sub$\mathrm{mm}$ source is seen to the north-west, with low-level extended sub-mm emission connecting the two.

G16.86-2.16 (18265-1517) This field (shown in Figs. 1ce and $\mathrm{cf}$ ) contains an extended sub-mm source comprising a compact bright source at the centre of the field and an extended arm to the north. The central peak is coincident with a methanol maser site. The maser site is linearly extended in the NW-SE direction. Wilking et al. (1990) report broad CO(21) line wings and interpret this as evidence for an outflow from this region. However, it is not clear as to the orientation of such an outflow.

G21.88+0.01 (18282-0951) This field (shown in Figs. 1cg and ch) contains a large HII region, which is coincident with the brightest sub-mm emission. The methanol maser site found to the north of this position is coincident with an unresolved sub-mm source.

G22.36+0.07 (18290-0924) This field (shown in Figs. 1ci and $\mathrm{cj}$ ) has a methanol maser site located at the centre. The maser site is coincident with the peak of sub-mm emission. A second peak is found to the south-west. The methanol maser site shows a linear feature stretching in the NW-SE direction.

G25.65+1.05 (18316-0602) This field (shown in Figs. 1ck and $\mathrm{cl}$ ) exhibits strong, extended sub-mm emission, which peaks at the position of an UC HII region and methanol maser site. This region has also been imaged at $350 \mu \mathrm{m}$ by Hunter et al. (2000), which shows a similar morphology to our map.

G23.26-0.24 (18317-0845) This field (shown in Figs. 1 cm and $\mathrm{cn}$ ) contains a methanol maser site at the centre which is close to, but not coincident with a peak in the extended sub-mm emission at $850 \mu \mathrm{m}$, however, the $450 \mu \mathrm{m}$ image (Fig. 1cn) indicates the maser site is coincident with a sub-mm peak. 
The sub-mm emission extends to the east and it appears there is stronger emission to the east of the imaged field. No methanol masers were detected at this eastern ridge.

G23.44-0.18(18319-0834) This field (shown in Figs. 1co and $\mathrm{cp}$ ) contains two methanol maser sites separated by 20 ". The sub-mm emission is extended in the north-south direction, similar to the orientation of the two maser sites, and suggests there may be two sub-mm sources associated with the two maser sites. Indeed, the $450 \mu \mathrm{m}$ image (Fig. 1co) does reveal two separate peaks, each within 4 " of the maser sites. The northern maser site has a linear morphology extending in the north-south direction. Forster \& Caswell (1999) report $\mathrm{OH}$ maser emission coincident with the two methanol maser sites. They also report water maser emission at the position of the southern methanol maser site, as well as a further water maser site located about $12^{\prime \prime}$ to the south-west.

G24.79+0.08 (18335-0711a) This field (shown in Figs. 1cq and cr) contains a strong, compact sub-mm source, with low level extended emission around it. A methanol maser site is located at the peak of the sub-mm emission. The linear morphology of the maser site extends in the NW-SE direction, similar to the orientation of the extended sub-mm continuum emission.

G24.85+0.09 (18335-0711b) This field (shown in Figs. 1cs and ct) contains extended sub-mm emission peaking at the position of a methanol maser site. A negative artefact is apparent in Fig. 1cs at the southern edge. This is caused by the emission from $\mathrm{G} 24.79+0.08$ being chopped into the image.

G25.71+0.04 (18353-0628) This field (shown in Figs. 1cu and cv) shows two distinct sub-mm peaks, as well as lower level extended emission. The brightest peak at the centre of the field is coincident with a methanol maser site, whilst the second, weaker sub-mm peak is close to an UC HII region.

G25.83-0.18(18361-0627) This field (shown in Figs. 1cw and cx) has a compact sub-mm source located at the centre of the image and coincident with a methanol maser site.

G28.15-0.00 (18403-0417a) This field (shown in Figs. 1cy and cz) includes a methanol maser site coincident with the peak of an extended sub-mm source.

G28.20-0.05 (18403-0417b) This field (shown in Figs. 1da and $\mathrm{db}$ ) had an extended sub-mm source at the centre, which peaks at the position of a methanol maser site and UC HII region.

G28.31-0.39 (18416-0420a) This field (shown in Figs. 1dc and dd) has extended sub-mm emission throughout most of the imaged region. A methanol maser site is found at the centre of the field and is coincident with a local peak of the sub-mm continuum emission. The sub-mm emission appears to be roughly extended in the east-west direction. Strong sub-mm emission is noticed at the western edge of the field, which is due to emission from G28.28-0.36 (Figs. 1de and df). Negative emission can be seen in the southern part of Figs. 1dc and dd, which is also due to chopped in emission from $\mathrm{G} 28.28-0.36$.

G28.28-0.36 (18416-0420b) This field (shown in Figs. 1de and df) contains bright, extended sub-mm emission, which is dominated by two peaks. One is close to a methanol maser site (towards the centre of the field) and the other is close to an UC HII region. The $450 \mu$ m image (Fig. 1df) has a peak of emission, which coincides with the maser site.

G28.85-0.23 (18421-0348a) This field (shown in Figs. $1 \mathrm{dg}$ and $\mathrm{dh}$ ) contains extended sub-mm emission. A sub-mm peak is seen close to the position of a methanol maser site in the centre of the image. A strong negative artefact is apparent in the south-western quarter of the image, which is due to chopped in emission from G28.83-0.25 (Figs. 1di and $\mathrm{dj})$.

G28.83-0.25 (18421-0348b) This field (shown in Figs. 1di and $\mathrm{dj}$ ) is dominated by an extended sub-mm source, whose peak coincides with a methanol maser site.

G29.98-0.05 (18434-0242a) This field (shown in Figs. 1dk and dl) has extended sub-mm emission peaking close to a methanol maser site.

G29.86-0.04 (18434-0242b) This field (shown in Figs. $1 \mathrm{dm}$ and $\mathrm{dn}$ ) exhibits extended sub-mm emission across the field. The strongest peak is found close to (but not coincident with) a methanol maser site, at the centre of the field.

G29.96-0.02 (18434-0242c) This field (shown in Figs. 1do and dp) contains extended sub-mm emission peaking at the centre of the field. Close to the peak, a methanol maser site and an UC HII region (separated from the maser site by $5^{\prime \prime}$ ) are found. The UC HII region has been studied in detail and is cometary in shape (Hofner \& Churchwell 1996), and powered by an O5.5 star (Wood \& Churchwell 1989b). At the position of the methanol maser site Hofner \& Churchwell (1996) also report water maser emission. This is also coincident with $\mathrm{NH}_{3}(4,4)$ emission, a tracer of hot cores (Cesaroni et al. 1998).

G30.82+0.27 (18440-0148a) This field (shown in Figs. 1dq and dr) contains an extended sub-mm source at the centre of the field, whose peak coincides with one of two methanol maser sites. The $450 \mu \mathrm{m}$ image (Fig. 1dr) has two peaks, corresponding to the elongated $850 \mu \mathrm{m}$ structure.

G30.79+0.20 (18440-0148b) This field (shown in Figs. 1ds and dt) contains an extended sub-mm source towards the centre of the region. A methanol maser site is found close to, but not coincident with, the peak of the sub-mm source.

G30.78+0.23 (18440-0148c) This field (shown in Figs. 1du and dv) contains extended sub-mm emission on the eastern side of the region. The peak of the sub-mm emission (towards the centre) is coincident with a methanol maser site. A negative artefact is seen in Figs. 1du and dv about $25^{\prime \prime}$ to the south-west of the maser site and is likely to be emission outside the region that has been chopped in.

G30.59-0.04 (18443-0210) This field (shown in Figs. 1dw and $\mathrm{dx}$ ) shows extended sub-mm emission peaking towards the centre of the region. The peak is close to a methanol maser site, but not coincident with it.

G30.90+0.16 (18446-0150) This field (shown in Figs. 1dy and $\mathrm{dz}$ ) contains a sub-mm source in the centre of the region, as well as lower level extended emission throughout the field. A methanol maser site is found coincident with the peak of the sub-mm emission.

G31.41+0.31 (18449-0115) This field (shown in Figs. 1ea and eb) shows a strong sub-mm source at the centre of the field. 
The peak of the sub-mm emission is close to a methanol maser site. An UC HII region is located about $20^{\prime \prime}$ to the north. Hofner \& Churchwell (1996) find that the radio continuum emission extends from the UC HII position to the maser position. The methanol maser site is also coincident with water maser emission (Hofner \& Churchwell 1996). The maser site is also coincident with the hot core tracer $\mathrm{NH}_{3}(4,4)$, detected by Cesaroni et al. (1998).

G30.82-0.05 (18450-0200b) This field (shown in Figs. 1ec and ed) contains two methanol maser sites, close to the centre of the region. They are also close to the peak of a sub-mm source which is elongated in the NE-SW direction.

G30.76-0.05 (18450-0200c) This field (shown in Figs. 1ee and ef) has a methanol maser site at the centre. Extended sub-mm emission is seen throughout the imaged region, with one peak (not the strongest) close to the maser site. There are at least four sub-mm peaks in the field.

G30.71-0.06 (18450-0200d) This field (shown in Figs. 1eg and eh) exhibits widespread sub-mm continuum emission. The strongest sub-mm peak is close to a methanol maser site. An UC HII region is located approximately $30^{\prime \prime}$ to the north of the maser site. Whilst it is found within the sub-mm emission contours, it does not appear to be near any local peak in the sub-mm emission.

G31.06+0.09 (18452-0141) This field (shown in Figs. 1ei and ej) has a weak sub-mm source towards the centre of the field. The peak of the sub-mm emission is close to, but not coincident with a methanol maser site.

G31.28+0.06 (18456-0129) This field (shown in Figs. 1ek and el) exhibits a sub-mm source towards the centrenedi of the field, with a methanol maser site close to, but not coincident with, the peak of the sub-mm emission. An UC HII region is located about $5^{\prime \prime}$ to the north-east. Whilst the UC HII region is within the sub-mm emission contours, it does not appear to be closely related to any local sub-mm maximum. The methanol maser site is coincident with $\mathrm{OH}$ and water maser sites (Forster \& Caswell 1999).

\section{Discussion}

The images in Fig. 1 reveal some interesting details. Most prominent is the fact that nearly every maser site (81 out of 84) is close to the peak of a sub-mm source. This very close association between maser sites and sub-mm peaks is remarkable in light of the fact that most of the maser sites do not show signs of an UC HII region - the signature of an embedded massive star.

Many of the images show multiple sub-mm sources. In such fields, it is likely that all the sub-mm sources belong to the same star forming cloud, allowing us to make some interpretations of the relative brightnesses of sources. There are examples where the sub-mm source associated with an UC HII region is brighter than the sub-mm source associated with a maser site - G6.54-0.11, G8.68+0.36, G21.88+0.01. This could be interpreted to mean that UC HII regions are typically more luminous than the powering sources of maser sites. However, there are also many examples where the sub-mm source associated with a maser site is brighter than the sub-mm source associated with an UC HII region - G9.99+0.03, G14.60+0.02, G19.49+0.15, $\mathrm{G} 25.71+0.04$. Such examples suggest maser sites can be at least as luminous as UC HII regions, and because no radio continuum is detected, they are likely to be in a stage of evolution before the UC HII region has been created.

The images show many fields where strong sub-mm sources are detected, but are not associated with either maser or radio continuum emission, such as G14.60+0.02. In this example there is a relatively weak sub-mm source associated with an UC HII region, and a stronger sub-mm source associated with a maser site, but the strongest sub-mm source in the field is not associated with either. Since the weakest sub-mm source is associated with an UC HII region, then we can assume that all three are luminous enough to create an UC HII region. Thus, the most likely explanation is that the brightest sub-mm source in this field is the youngest, at an evolutionary stage not only before the UC HII region has turned on, but also before the maser emission has turned on.

\subsection{Association of methanol masers and molecular gas}

Other surveys have shown the close correlation between dense gas traced by molecular line emission and embedded massive stars traced by IRAS sources (e.g. Bronfman et al. 1996; Molinari et al. 1996). Here we demonstrate the close association of dense molecular gas tracers and methanol maser sites. Table 2 lists details of detections of molecular line emission whose positional uncertainties overlap with the positions of methanol maser sites. Since the maser sites lie close to the Galactic plane, it is not surprising that we find many coincidences. Thus, we also provide radial velocities for both the molecular lines and methanol masers. Out of 50 maser sites coincident with known dense gas, $80 \%$ have radial velocities within $5 \mathrm{~km} \mathrm{~s}^{-1}$ of the dense gas radial velocity, showing the association of dense gas and methanol masers.

\subsection{Bolometric luminosities}

A simple analysis of these data can be made by estimating the bolometric luminosity of some sources. Table 3 lists bolometric luminosities of some sources obtained from the literature. We can also derive bolometric luminosities using our SCUBA fluxes. To do this, we require some information on the SED of each source, other than the two SCUBA points at 450 and $850 \mu \mathrm{m}$. For this purpose, we have correlated the positions of IRAS and MSX point sources. We consider a SCUBA source associated with an IRAS source if the IRAS error ellipse overlaps the SCUBA source. A SCUBA source is considered associated with a MSX source if the peak of the sub-mm emission is within 6" of the peak of the MIR emission - the pixel size of the MSX images. To evaluate the MIR fluxes, we referred directly to the MSX images, rather than MSX Point Source Catalog (PSC). This is because we found occasional discrepancies between MSX PSC fluxes and those derived from the MSX images. For example, the MSX PSC quotes upper limits to the 8 and $12 \mu \mathrm{m}$ fluxes of 0.085 and $1.3 \mathrm{Jy}$, respectively, 
Table 2. Comparison of molecular gas and methanol maser kinematics. Column 1 lists the sub-mm source detected in this paper. Columns $2-4$ give the name, radial velocity and reference for the associated molecular transition to the sub-mm source, respectively. See footnote for details of references. Column 5 gives the radial velocity range over which Walsh et al. (1998) detected methanol maser emission. One number is given for maser sites where only one maser spot was detected. Column 6 gives adopted distances from the literature, with references given in Col. 7. Those distances marked with an asterisk (*) were derived assuming $10 \mathrm{kpc}$ to the Galactic centre; all others assume it is $8.5 \mathrm{kpc}$ away. The distance to the source marked with a double-asterisk $\left(^{* *}\right)$ was not determined using the kinematics of a Galactic rotation curve. Columns 8 and 9 list bolometric luminosities and references for sources where available, respectively.

\begin{tabular}{|c|c|c|c|c|c|c|c|c|}
\hline $\begin{array}{l}\text { Source } \\
\text { name }\end{array}$ & $\begin{array}{l}\text { Molecular } \\
\text { line } \\
\text { transition }\end{array}$ & $\begin{array}{c}\text { Radial } \\
\text { velocity } \\
\left(\mathrm{km} \mathrm{s}^{-1}\right)\end{array}$ & Ref. & $\begin{array}{c}\text { Methanol } \\
\text { maser velocity } \\
\left(\mathrm{km} \mathrm{s}^{-1}\right)\end{array}$ & $\begin{array}{c}\text { Distance } \\
(\mathrm{kpc})\end{array}$ & Ref. & $\begin{array}{c}\text { Luminosity } \\
10^{4} L_{\odot}\end{array}$ & Ref. \\
\hline G0.21-0.00 & $\mathrm{C}^{34} \mathrm{~S}(2-1)$ & -2.7 & $(1)$ & $49.4-42.1$ & 7.36 & (1) & & \\
\hline $\mathrm{G} 0.83+0.18$ & $\mathrm{CS}(2-1)$ & +5.4 & (2) & $3.5-2.4$ & $5.0 / 12$ & $(25)$ & & \\
\hline G0.32-0.20 & CS (2-1) & +18.7 & (2) & $26.5-14.6$ & $8.1 / 8.9$ & (25) & & \\
\hline G0.55-0.85 & $\operatorname{CS}(7-6)$ & +16.6 & (3) & $10.8-19.3$ & $2.0^{*}$ & (3) & & \\
\hline G5.90-0.43 & & & & $9.2-10.1$ & $2.6^{*}$ & (4) & & \\
\hline $\mathrm{G} 8.14+0.23$ & $\mathrm{CS}(2-1)$ & +19.4 & (2) & 5.0 & $4.2^{*}$ & (5) & 19 & (5) \\
\hline G10.10+0.73 & $\mathrm{CO}(1-0)$ & +38.6 & (6) & 1.2 & $0.69 / 16$ & (25) & & \\
\hline G9.62+0.19 & $\mathrm{CS}(2-1)$ & +5.0 & (2) & $-2.1-6.3$ & $0.70 / 16$ & (25) & & \\
\hline G8.68-0.36A & $\mathrm{SiO}(2-1)$ & +37.2 & (7) & 39.0 & 4.5 & (7) & & \\
\hline G8.68-0.36B & $\mathrm{SiO}(2-1)$ & +37.2 & (7) & $40.9-44.0$ & 4.5 & (7) & & \\
\hline G9.99-0.03A & $\mathrm{CS}(2-1)$ & +48.7 & (2) & $40.8-47.9$ & 5.0 & (8) & 3.0 & (8) \\
\hline G10.30-0.15A & $\mathrm{CS}(5-4)$ & +13.6 & (9) & 20.0 & $6.0^{*}$ & (4) & & \\
\hline G10.34-0.14A & $\mathrm{CS}(5-4)$ & +13.6 & (9) & $14.3-16.7$ & $6.0^{*}$ & (4) & & \\
\hline G10.32-0.16 & $\mathrm{CS}(5-4)$ & +13.6 & (9) & $9.9-13.0$ & $6.0^{*}$ & (4) & & \\
\hline G10.29-0.13A & $\mathrm{CS}(5-4)$ & +13.6 & (9) & $2.2-7.8$ & $6.0^{*}$ & (4) & & \\
\hline G10.63-0.38 & CS (7-6) & -2.2 & (3) & $2.2-5.9$ & 6.0 & (10) & & \\
\hline G12.89+0.49 & $\mathrm{CS}(2-1)$ & +33.8 & (2) & $30.0-39.2$ & 3.4 & (8) & 6.3 & (8) \\
\hline G12.03-0.03 & $\mathrm{CS}(2-1)$ & +109.8 & $(2)$ & 107.5 & $6.7 / 10$ & (25) & & \\
\hline G11.94-0.15B & $\mathrm{CS}(2-1)$ & +13.3 & (2) & $47.5-48.3$ & $4.8 / 11.8$ & (25) & & \\
\hline G12.21-0.09A & $\mathrm{SiO}(2-1)$ & +24.4 & (7) & 19.7 & $3.1 / 13.5$ & $(7) /(11)$ & $100(13.5 \mathrm{kpc})$ & (4) \\
\hline G12.21-0.09B & $\mathrm{SiO}(2-1)$ & +24.4 & $(7)$ & $17.0-20.5$ & $3.1 / 13.5$ & $(7) /(11)$ & & \\
\hline G12.21-0.09C & $\mathrm{SiO}(2-1)$ & +24.4 & (7) & 67.9 & $3.1 / 13.5$ & $(7) /(11)$ & & \\
\hline G11.94-0.62A & $\mathrm{CS}(2-1)$ & +39.7 & (12) & $30.2-43.4$ & 4.2 & (11) & & \\
\hline G12.68-0.18A & $C^{34} S(2-1)$ & +58.1 & (13) & $51.3-58.5$ & 4.9 & (13) & 0.44 & (13) \\
\hline \multirow[t]{3}{*}{ G12.91-0.26 } & $\mathrm{CS}(2-1)$ & +36.6 & $(2)$ & $35.2-39.9$ & 3.7 & (13) & 13 & (13) \\
\hline & & & & & & & 17 & (5) \\
\hline & & & & & & & 11 & (14) \\
\hline G11.50-1.49 & $\mathrm{CS}(2-1)$ & +10.6 & (2) & $6.3-16.2$ & 1.8 & (7) & & \\
\hline $\mathrm{G} 14.60+0.02 \mathrm{~B}$ & $\mathrm{CS}(2-1)$ & +24.4 & (2) & $23.3-25.5$ & 2.8 & (7) & & \\
\hline \multirow[t]{2}{*}{ G15.03-0.68B } & $\mathrm{CS}(5-4)$ & +19.5 & (9) & $21.1-23.2$ & $1.3^{* *}$ & (15) & 43 & (16) \\
\hline & & & & & & & 0.48 & (17) \\
\hline G16.59-0.05 & $\mathrm{CS}(2-1)$ & +59.1 & (2) & $57.0-68.6$ & 4.7 & (18) & & \\
\hline G19.49+0.15 & $\mathrm{CS}(2-1)$ & +24.1 & $(2)$ & $20.9-24.2$ & 2.0 & (7) & & \\
\hline G19.36-0.03 & $\mathrm{CS}(2-1)$ & +25.9 & $(2)$ & $24.7-28.7$ & 2.5 & $(8)$ & 1.0 & (8) \\
\hline G19.61-0.13A & $\mathrm{CS}(1-0)$ & +59.7 & (10) & $50.2-56.2$ & $4.5^{*}$ & (3) & & \\
\hline G19.61-0.13B & $\mathrm{CS}(1-0)$ & +59.7 & (10) & 52.4 & $4.5^{*}$ & (3) & & \\
\hline G16.86-2.16A & $\mathrm{CH}_{2} \mathrm{O}\left(1_{10}-1_{11}\right)$ & +18.0 & (19) & $14.8-18.6$ & 2.1 & (19) & 0.71 & (20) \\
\hline $\mathrm{G} 21.88+0.01 \mathrm{~B}$ & $\mathrm{CS}(2-1)$ & +22.4 & (2) & 20.6 & $2.0 / 13.7$ & (25) & & \\
\hline $\mathrm{G} 22.36+0.07 \mathrm{~A}$ & $\mathrm{CS}(2-1)$ & +84.3 & $(2)$ & $77.0-85.0$ & $12.2^{*}$ & (3) & & \\
\hline G25.65+1.05 & CS (2-1) & +41.4 & (2) & $40.8-42.4$ & 3.2 & (8) & 4.1 & (8) \\
\hline G23.44-0.18 & CS (2-1) & +104.2 & (2) & $95.7-107.3$ & $9.0^{*}$ & (4) & & \\
\hline $\mathrm{G} 24.79+0.08$ & $\mathrm{SiO}(2-1)$ & +110.5 & $(7)$ & $106.7-114.6$ & 7.7 & (18) & 3.3 & (18) \\
\hline $\mathrm{G} 25.71+0.04 \mathrm{~A}$ & $\mathrm{H} 76 \alpha$ & +103.0 & $(21)$ & $89.8-100.0$ & $14.0^{*}$ & (4) & 59 & (4) \\
\hline G28.20-0.05 & CS (2-1) & +97.4 & $(2)$ & 101.0 & $9.1^{*}$ & (22) & 48 & (22) \\
\hline
\end{tabular}


Table 2. continued.

\begin{tabular}{|c|c|c|c|c|c|c|c|c|}
\hline $\begin{array}{l}\text { Source } \\
\text { name }\end{array}$ & $\begin{array}{l}\text { Molecular } \\
\text { line } \\
\text { transition }\end{array}$ & $\begin{array}{c}\text { Radial } \\
\text { velocity } \\
\left(\mathrm{km} \mathrm{s}^{-1}\right)\end{array}$ & Ref. & $\begin{array}{c}\text { Methanol } \\
\text { maser velocity } \\
\left(\mathrm{km} \mathrm{s}^{-1}\right)\end{array}$ & $\begin{array}{c}\text { Distance } \\
(\mathrm{kpc})\end{array}$ & Ref. & $\begin{array}{c}\text { Luminosity } \\
10^{4} L_{\odot}\end{array}$ & Ref. \\
\hline G28.28-0.36B & & & & 40.4 & $3.3^{*}$ & (22) & & \\
\hline G28.85-0.23 & & & & 99.8 & $6.3^{*}$ & (3) & & \\
\hline G28.83-0.25 & $\mathrm{CS}(2-1)$ & +87.3 & (13) & 81.0-91.7 & $6.3^{*}$ & (3) & & \\
\hline G29.96-0.02A & CS (2-1) & +97.4 & (2) & $95.7-100.1$ & 9.0 & (11) & 200 & (4) \\
\hline $\mathrm{G} 30.82+0.27$ & $\mathrm{CS}(2-1)$ & +97.6 & (2) & $104.6-110.1$ & 8.2 & (13) & 4.7 & (13) \\
\hline G30.59-0.04 & & & & 43.2 & $14.1^{*}$ & (3) & & \\
\hline \multirow[t]{2}{*}{$\mathrm{G} 31.41+0.31$} & CS (2-1) & +96.6 & (2) & $92.4-105.0$ & 7.9 & (23) & 22 & (23) \\
\hline & & & & & & & 20 & (24) \\
\hline G30.82-0.05 & CS (2-1) & +95.1 & (2) & $92.8-108.2$ & 6.6 & (7) & & \\
\hline $\mathrm{G} 31.28+0.06$ & CS (2-1) & +109.9 & (2) & $104.3-112.0$ & $7.2^{*}$ & (22) & & \\
\hline
\end{tabular}

References - (1) Juvela (1996), (2) Bronfman et al. (1996), (3) Plume et al. (1992), (4) Wood \& Churchwell (1989b), (5) Chini et al. (1986a), (6) Shepherd \& Churchwell (1996), (7) Harju et al. (1998), (8) Molinari et al. (1996), (9) Olmi \& Cesaroni (1999), (10) Anglada et al. (1996), (11) Hofner \& Churchwell (1996), (12) Churchwell et al. (1992), (13) Larionov et al. (1999), (14) Gürtler et al. (1991), (15) Hanson et al. (1997), (16) Chini et al. (1986b), (17) Nielbock et al. (2001), (18) Codella et al. (1997), (19) MacLeod et al. (1998), (20) Hughes \& MacLeod (1993), (21) Kim \& Koo (2001), (22) Kurtz et al. (1994), (23) Cesaroni et al. (1998), (24) Hatchell et al. (2000), (25) this paper.

Table 3. Estimated bolometric luminosities of sub-mm sources. Each source shown in Fig. 2, with a known distance and an IRAS and/or MSX counterpart is listed. The adopted distance in Col. 2 is either from Table 1 (corrected for $R_{0}=8.5 \mathrm{kpc}$ and $\Theta_{0}=220 \mathrm{~km} \mathrm{~s}^{-1}$ ) or derived using the methanol maser radial velocity and the Galactic rotation model of Brand \& Blitz (1993). The derived bolometric luminosity of each source is given in Col. 3. Associations are shown in Col. 4, with the same notation used as Table 1 .

\begin{tabular}{cccc}
\hline \hline $\begin{array}{c}\text { Source } \\
\text { name }\end{array}$ & $\begin{array}{c}\text { Adopted } \\
\text { distance } \\
(\mathrm{kpc})\end{array}$ & $\begin{array}{c}\text { Bolometric } \\
\text { luminosity } \\
\left(10^{4} L_{\odot}\right)\end{array}$ & Assoc. \\
\hline G8.14+0.23B & 3.6 & 13 & $\mathrm{mr}$ \\
G10.63-0.38 & 6.0 & 84 & $\mathrm{mr}$ \\
G11.50-1.49 & 1.8 & 0.69 & $\mathrm{~m}$ \\
$\mathrm{G} 11.94-0.62 \mathrm{~A}$ & 4.2 & 11 & $\mathrm{mr}$ \\
$\mathrm{G} 12.03-0.03$ & 6.7 & 1.6 & $\mathrm{~m}$ \\
$\mathrm{G} 12.89+0.49$ & 3.4 & 3.9 & $\mathrm{~m}$ \\
$\mathrm{G} 12.91-0.26$ & 3.7 & 10 & $\mathrm{~m}$ \\
$\mathrm{G} 21.88+0.01 \mathrm{~B}$ & 2.0 & 0.58 & $\mathrm{~m}$ \\
$\mathrm{G} 28.20-0.05$ & 7.7 & 32 & $\mathrm{mr}$ \\
$\mathrm{G} 30.82+0.27$ & 8.2 & 5.1 & $\mathrm{~m}$ \\
\hline
\end{tabular}

for the source G0.55-0.85. However, a MIR source well above these limits is clearly seen in the images. We notice that the region in question shows many sources close together, as well as extended emission. So we consider confusion as a likely cause for such errors in the MSX PSC. To calculate the fluxes of each source in the four MSX bands, we integrated the emission in the image over the source, and then removed a sky value, determined from an appropriately chosen nearby part of the image. The SEDs of those SCUBA sources associated with an
IRAS and/or MSX source are shown in Fig. 2. To avoid contamination of IRAS fluxes, we did not include IRAS points in our SEDs when another SCUBA source was seen within one arcminute of the IRAS position.

It has been established (Walsh et al. 1999) that a simple polynomial fit to the SED of an embedded massive star can be reliably used to estimate the bolometric luminosity of the star to within an order of magnitude, even though such a fit does not accurately match the detailed features of the spectrum, such as silicate absorption features or dust emissivity. This is because the SED is dominated by the emission from dust around the star which re-radiates most of the energy as an approximate blackbody, peaking around $100 \mu \mathrm{m}$. Thus, we have fitted 5th order polynomials to each source shown in Fig. 2.

We have integrated under the curve to derive the bolometric luminosity, which is listed in Table 3. G0.50+0.19 is omitted from the table as its distance is unknown, and thus its luminosities could not be determined. The distances to the remaining sources are adopted either from Table 2 or derived from the kinematic distances using the Galactic rotation curve of Brand \& Blitz (1993), which assume $R_{0}=8.5 \mathrm{kpc}$ and $\Theta_{0}=220 \mathrm{~km} \mathrm{~s}^{-1}$.

We note that all sources in Table 3 have high bolometric luminosities $\left(L \geq 0.58 \times 10^{4} L_{\odot}\right)$, consistent with single stars of spectral type B3 or earlier. Since over half of the sources in Table 3 are associated with methanol maser sites and given that they all have such high luminosities, we confirm the results of Walsh et al. (2001) that many maser sources are associated with very young high-mass stars.

\section{Conclusions}

Our survey of the $450 / 850 \mu$ m emission from dust in the fields of 73 methanol masers in the declination range $0>\delta>-20$ has included within these fields a total of 84 methanol maser 


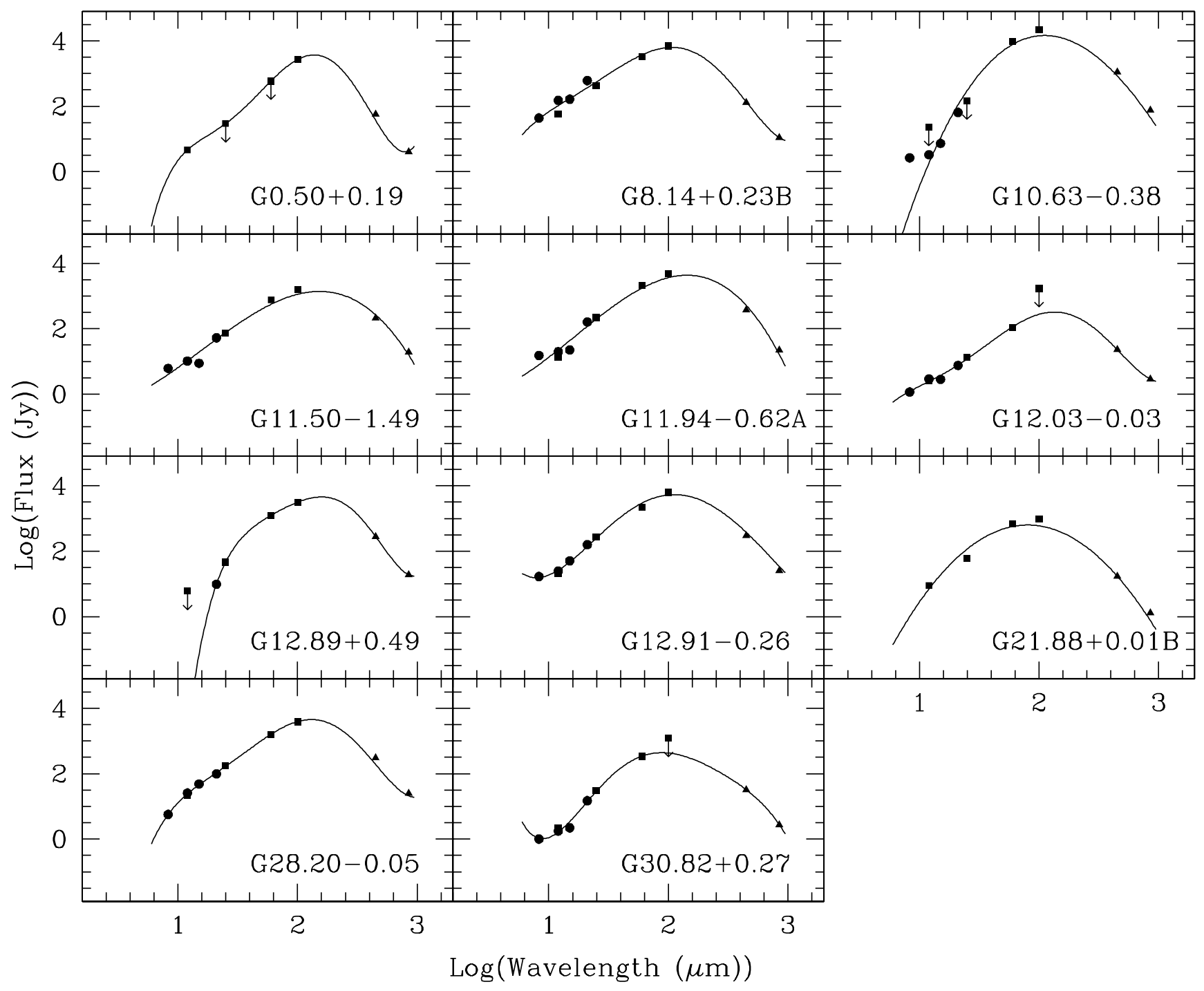

Fig. 2. Spectral energy distributions of SCUBA sources associated with IRAS and/or MSX sources. SCUBA data are represented by filled triangles, IRAS data are represented by filled squares and MSX data are represented by filled circles. Upper limits to any data point are represented by downward pointing arrows. The curves represent fifth order polynomial fits to the datapoints. These fits are used to determine the bolometric luminosities of each source by integrating under the curve between $8 \mu \mathrm{m}$ and $850 \mu \mathrm{m}$.

sites and 30 radio continuum sources and yielded the following results:

1. Most methanol maser sites (81 out of 84 ) are within $10^{\prime \prime}$ of a sub-mm peak. All except one of the maser sites coincide with sub-mm continuum emission from dust, confirming that the methanol maser emission arises from deeply embedded objects.

The one exception is $\mathrm{G} 10.10+0.73$, where the maser lies $90^{\prime \prime}$ away from a compact sub-mm and radio continuum source coincident with the bipolar planetary nebula NGC 6537. Since no emission was detected at $450 \mu \mathrm{m}$ from NGC 6537, it is possible that the sub-mm emission detected is free-free emission from ionised gas rather than continuum radiation from dust. It may be significant that this field contains the only planetary nebula and the only maser site with no associated sub-mm emission in our sample. A subsequent one-hour integration at the maser position, with a $1 \sigma$ noise level of $8 \mathrm{mJy}_{\text {beam }}{ }^{-1}$ at $850 \mu \mathrm{m}$, failed to reveal any sub-mm flux. In this case it is not clear whether the association between the maser site and the planetary nebula is coincidental. This may be a unique example where methanol maser emission is not associated with massive star formation.

2. Many of the fields mapped show dust emission from complex structures, with extended emission coincident with the maser linked to other clumps and spurs. There are several examples of separate, isolated clumps apparently unconnected with the central core with no associated methanol maser source. These "maserless cores" are most interesting since the absence of maser emission may indicate a lower methanol abundance and/or lower excitation, suggesting that they may harbour condensed objects at an earlier evolutionary stage than their masing counterparts. Further investigation of their nature should be undertaken. 
3. The SEDs for those sources for which distance measurements are available yield bolometric luminosities in excess of $10^{3} L_{\odot}$. This confirms the results of Walsh et al. (2001) that methanol maser sources are good indicators of highmass star formation.

Acknowledgements. NDSA thanks PPARC for studentship support and MGB and J-KL acknowledge financial support from the ARC (Australian Research Council) and ANSTO (Australian Nuclear Science and Technology Organisation) - Access to Major Research Facilities Program, while this research was being carried out. The James Clerk Maxwell Telescope is operated by the Joint Astronomy Centre, Hawaii, on behalf of the UK PPARC, the Netherlands NWO and the Canadian NRC.

\section{References}

Acker, A., Chopinet, M., Pottasch, S. R., \& Stenholm, B. 1987, A\&AS, 71, 163

Alvey, N. D. S. A. 2001, Ph.D. Thesis, University of Kent, UK

Anglada, G., Estalella, R., Pastor, J., Rodríguez, L. F., \& Haschick, A. D. 1996, ApJ, 463, 205

Brand, J., \& Blitz, L. 1993, A\&A, 275, 67

Bronfman, L., Nyman, L-A., \& May, J. 1996, A\&AS, 115, 81

Caswell, J. L., Vaile, R. A., \& Forster, J. R. 1995, MNRAS, 277, 210

Caswell, J. L. 1998, MNRAS, 297, 215

Cesaroni, R., Churchwell, E., Hofner, P., Walmsley, C. M., \& Kurtz, S. 1994, A\&A, 288, 903

Cesaroni, R., Hofner, P., Walmsley, C. M., \& Churchwell, E. 1998, A\&A, 331, 709

Chini, R., Kreysa, E., Mezger, P. G., \& Gemuend, H.-P. 1986, A\&A, 154, L8

Chini, R., Kreysa, E., Mezger, P. G., \& Gemuend, H.-P. 1986, A\&A, $157, \mathrm{~L} 1$

Churchwell, E., Walmsley, C. M., \& Wood, D. O. S. 1992, A\&A, 253, 541

Codella, C., Testi, L., \& Cesaroni, R. 1997, A\&A, 325, 282

Forster, J. R., \& Caswell, J. L. 1999, A\&AS, 137, 43

Forster, J. R., \& Caswell, J. L. 2000, ApJ, 530, 371

Gürtler, J., Henning, Th., Krügel, E., \& Chini, R. 1991, A\&A, 252, 801

Hanson, M. M., Howarth, I. D., \& Conti, P. S. 1997, ApJ, 489, 698

Harju, J., Lehtinen, K., Booth, R. S., \& Zinchenko, I. 1998, A\&AS, 132,211

Hatchell, J., Fuller, G. A., Millar, T. J., Thompson, M. A., \& Macdonald, G. H. 2000, A\&A, 357, 637

Hobson, M. P., Padman, R., Scott, P. F., Prestage, R. M., \& Ward-Thompson, D. 1993, MNRAS, 264, 1025
Hofner, P., \& Churchwell, E. 1996, A\&AS, 120, 283

Hofner, P., Kurtz, S., Churchwell, E., Walmsley, C. M., \& Cesaroni, R. 1996, ApJ, 460, 359

Hofner, P., Wiesemeyer, H., \& Henning, T. 2001, ApJ, 549, 425

Holland, W. S., Robson, E. I., Gear, W. K., et al. 1999, MNRAS, 303, 659

Hughes, V. A., \& MacLeod, G. C. 1993, AJ, 105, 1495

Hunter, T. R., Churchwell, E., Watson, C., et al. 2000, AJ, 119, 2711

Jenness, T., Scott, P. F., \& Padman, R. 1995, MNRAS, 276, 1024

Jenness, T., \& Lightfoot, J. F. 1998, in Astronomical Data Analysis Software and Systems, ed. R. Albrecht, R. N. Hook, \& H. A. Bushouse, ASP Conf. Ser., 145, 216

Juvela, M. 1996, A\&AS, 118, 191

Kim, K.-T., \& Koo, B.-C. 2001, ApJ, 549, 979

Kurtz, S., Churchwell, E., \& Wood, D. O. S. 1994, ApJS, 91, 659

Larionov, G. M., Val'tts, I. E., Winnberg, A., et al. 1999, A\&AS, 139, 257

Maciel, W. J., \& Dutra, C. M. 1992, A\&A, 262, 271

MacLeod, G. C., Scalise Jr., E., Saedt, S., Galt, J. A., \& Gaylard, M. J. 1998, ApJ, 116, 1897

Menten, K. M., Walmsley, C. M., Henkel, C., et al. 1986, A\&A, 169, 271

Menten, K. M., Reid, M. J., Moran, J. M., et al. 1988, ApJ, 333, L83

Menten, K. M. 1991, ApJ, 380, L75

Minier, V., Booth, R. S., \& Conway, J. E. 1998, A\&A, 336, L5

Molinari, S., Brand, J., Cesaroni, R., \& Palla, F. 1996, A\&A, 308, 573

Nielbock, M., Chini, R., Jütte, M., \& Manthey, E. 2001, A\&A, 377, 273

Norris, R. P., Caswell, J. L., Wellington, K. J., McCutcheon, W. H., \& Reynolds, J. E. 1988, Nature, 335, 149

Norris, R. P., Whiteoak, J. B., Caswell, J. L., Wieringa, M. H., \& Gough, R. G. 1993, ApJ, 412, 222

Olmi, L., \& Cesaroni, R. 1999, A\&A, 352, 266

Phillips, C. J., Norris, R. P., Ellingsen, S. P., \& McCulloch, P. M. 1998, MNRAS, 300, 1131

Plambeck, R. L., \& Menten, K. M. 1990, ApJ, 364, 555

Plume, R., Jaffe, D. T., \& Evans II, N. J. 1992, ApJ, 78, 505

Shepherd, D. S., \& Churchwell, E. 1996, ApJ, 457, 267

Walsh, A. J., Hyland, A. R., Robinson, G., \& Burton, M. G. 1997, MNRAS, 291, 261

Walsh, A. J., Burton, M. G., Hyland, A. R., \& Robinson, G. 1998, MNRAS, 301, 640

Walsh, A. J., Burton, M. G., Hyland, A. R., \& Robinson, G. 1999, MNRAS, 309, 905

Walsh, A. J., Bertoldi, F., Burton, M. G., \& Nikola, T. 2001, MNRAS, 326, 36

Wilking, B. A., Blackwell, J. H., \& Mundy, L. G. 1990, AJ, 100, 758

Wood, D. O. S., \& Churchwell, E. 1989, ApJ, 340, 265

Wood, D. O. S., \& Churchwell, E. 1989, ApJS, 69, 831 